\title{
The Positioning of Sun Shadow \\ Jingyao Wang
}

School of Electric Power Engineering, North China Electric Power University, Baoding 071000, China.

763491449@qq.com

Keywords: positioning, length of sun shadow, variance, loop iteration

\begin{abstract}
The sun's shadow positioning technology is a method of determining the video filming location, through the analysis of object's shadow change in the video. After consulting relevant literature, we get the mathematical model for positioning by using the shadow of object, and predict the location in the example.

Firstly, we establish the function of shadow's length and the relevant variable. We get the mathematical model about the parameters by the formula derivation.

Secondly, by dealing with the data, we get the sun's shadow length of straight rod (once every 3 min test).Using the shadow length data after processing, we preliminarily determine the location of the longitude.

Finally, using the shadow length change model, through the loop iteration method, using the condition of fixed length, we calculate the variance of rod long to identify the probable place of measuring the length.
\end{abstract}

\section{Introduction}

How to determine the location of the video is one of the most important aspects of the video data analysis. The sun's shadow positioning technology is a method of determining the video filming location, through the analysis of object's shadow change in the video. On the basis of existing research, we solve the place by analyzing object's length of sun shadow. It has important research significance.

\section{Notations}

\begin{tabular}{cc}
\hline Symbols & Notation \\
\hline $\mathrm{l}$ & the shadow length of straight rod \\
$\mathrm{h}$ & the height of straight rod \\
$\varphi$ & latitude \\
$\delta$ & the sun declination \\
$\mathrm{t}$ & hour angle \\
$\lambda$ & longitude \\
$\mathrm{N}$ & days \\
$\mathrm{t}_{0}$ & Beijing time \\
\hline
\end{tabular}

\section{Model}

The mathematical model of the length of the shadow.

According to the geography knowledge and geometry relations, we can get the relation $[1,2]$ :

$$
\begin{gathered}
l=h / \tan (\arcsin (\sin \varphi \sin \delta+\cos \varphi \cos \delta \cos t)) \\
t=|12-[t 0-4 \times(120-\lambda) / 60]| \times 15 / 180 \times p i
\end{gathered}
$$




$$
\begin{gathered}
\delta=0.006918-0.399912 \cos b+0.0070257 \sin b-0.006758 \cos 2 b \\
+0.000907 \sin 2 b-0.002697 \cos 3 b+0.00148 \sin 3 b \\
\mathrm{pi}=3.1415926 \\
b=2 p i \times(N-1) / 365
\end{gathered}
$$

\section{The applying of the model to solving practical problem.}

In the case of known the sun shadow vertex coordinate data, we use the shadow length change model of multiple parameters to solve the probable places of the several straight rods [3].

Through the data processing of the known vertex coordinate data, we get the length data on April 18, 2015, as shown in Tab 1.

Tab 1 the length data and time

\begin{tabular}{|c|c|c|c|}
\hline $\mathrm{t}_{0}$ & $\mathrm{l}$ & $\mathrm{t}_{0}$ & $\mathrm{l}$ \\
\hline $14: 42$ & 1.1496 & $15: 15$ & 1.5402 \\
\hline $14: 45$ & 1.1822 & $15: 18$ & 1.5799 \\
\hline $14: 48$ & 1.2153 & $15: 21$ & 1.6201 \\
\hline $14: 51$ & 1.2491 & $15: 24$ & 1.6613 \\
\hline $14: 54$ & 1.2832 & $15: 27$ & 1.7033 \\
\hline $14: 57$ & 1.3180 & $15: 30$ & 1.7462 \\
\hline $15: 00$ & 1.3534 & $15: 33$ & 1.7901 \\
\hline $15: 03$ & 1.3894 & $15: 36$ & 1.8350 \\
\hline $15: 06$ & 1.4262 & $15: 39$ & 1.8809 \\
\hline $15: 09$ & 1.4634 & $15: 42$ & 1.9279 \\
\hline $15: 12$ & 1.5015 & \multicolumn{2}{c}{} \\
\cline { 1 - 3 } & & &
\end{tabular}

To observe the changing rule of the length of the shadow, we make the shadow length along with the change of time curve, as shown in Fig 1.

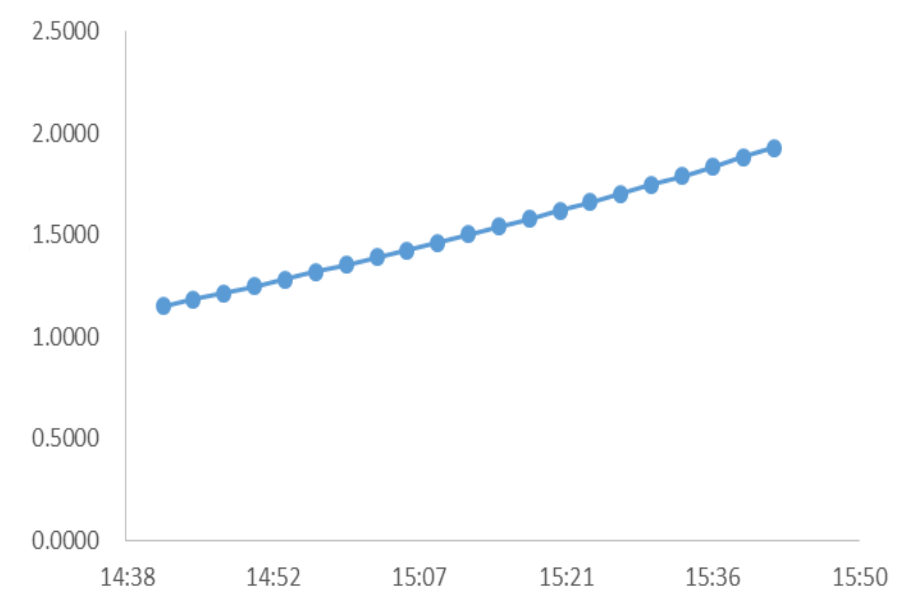

Fig 1 the shadow length change along the change of time curve

From the diagram, we can know that the length of the shadow is gradually increasing over time.

We use the known data mapping, finding the shadow changes should decline before 12:00 local time, then increasing, as shown in Fig 2. 


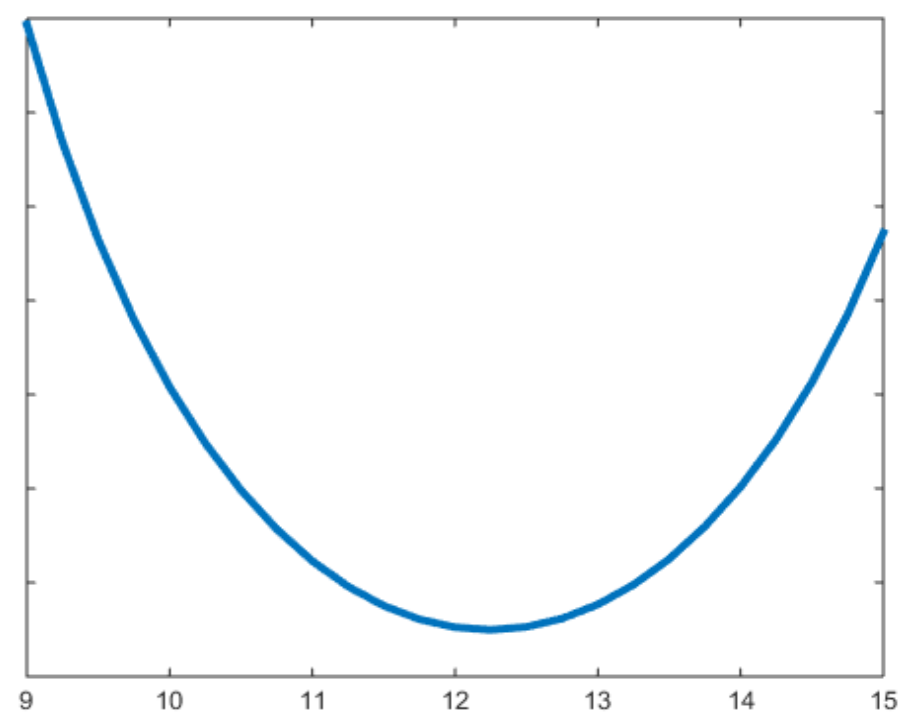

Fig 2 the tendency of the shadow length over time

Therefore, Beijing time 14:42-15:42 should be after 12:00 p.m. local time. We determine the area is located in east longitude 79 degrees east of 30 points.

The date of measurement date is April 18, 2015. So the date of the day $\mathrm{N}=31 \times 2+28+18=108$.

Combined with the data from the table 1, we build models [4]:

$$
s=\min \sqrt{\sum_{i=1}^{21}\left(h_{i}-\bar{h}\right)^{2}}
$$

We use iterative algorithm, generating the longitude and latitude of narrow range into formula. And we limit the mean square deviation s of straight rod height $\mathrm{h}$ to a small range $(\mathrm{s}<0.001)$. Thus get the satisfied condition of latitude and longitude, as shown in Tab 2.

Tab 2. the result of the longitude and latitude

\begin{tabular}{|c|c|}
\hline $100^{\circ} \mathrm{E}, 21^{\circ} \mathrm{N}$ & Myanmar \\
\hline $103^{\circ} \mathrm{E}, 20^{\circ} \mathrm{N}$ & Laos \\
\hline $105^{\circ} \mathrm{E}, 19^{\circ} \mathrm{N}$ & Vietnam \\
\hline $107^{\circ} \mathrm{E}, 18^{\circ} \mathrm{N}$ & Near the Xisha Islands \\
\hline
\end{tabular}

\section{Conclusions}

We compare the probable result, finding that all meet the basic requirements of the model whether long shadow changes or residual sum of squares. We look up the relevant information of four locations, to prove the validity of the solved locations. Thus, the method of comparing the length of sun shadow and screening the position by the sum of squared residuals is feasible.

\section{References}

[1]http://baike.baidu.com/link?url=VVzyl4YznA4GNzqHXxEXas_rWfho0r-YdIQeDh8qBo-fHJf87 ASzejDij-TdL_xTn8USv-shhPvr1pGPxsQBTa

[2] Guoan Wang, Hongtao Mi, Tianhong Deng, Yanan Li, Lanxia Li. Angle of the sun and solar azimuth sunrise and sunset moment a year range calculation [J]. Meteorology and environmental science, 2007, S1:161-164.

[3] Youhua Qin. Geometry analysis Angle of the sun [J]. The test (college entrance examination liberal arts version), 2010, Z1:72-74.

[4] Yong Peng, Junfeng Niu, Po Huo. MATLAB nonlinear regression model parameters [J]. Chemistry Bulletin, 2007, 11:880-883. 